\title{
Article
}

\section{Multi-redox Molecule for High-Energy Redox Flow Batteries}

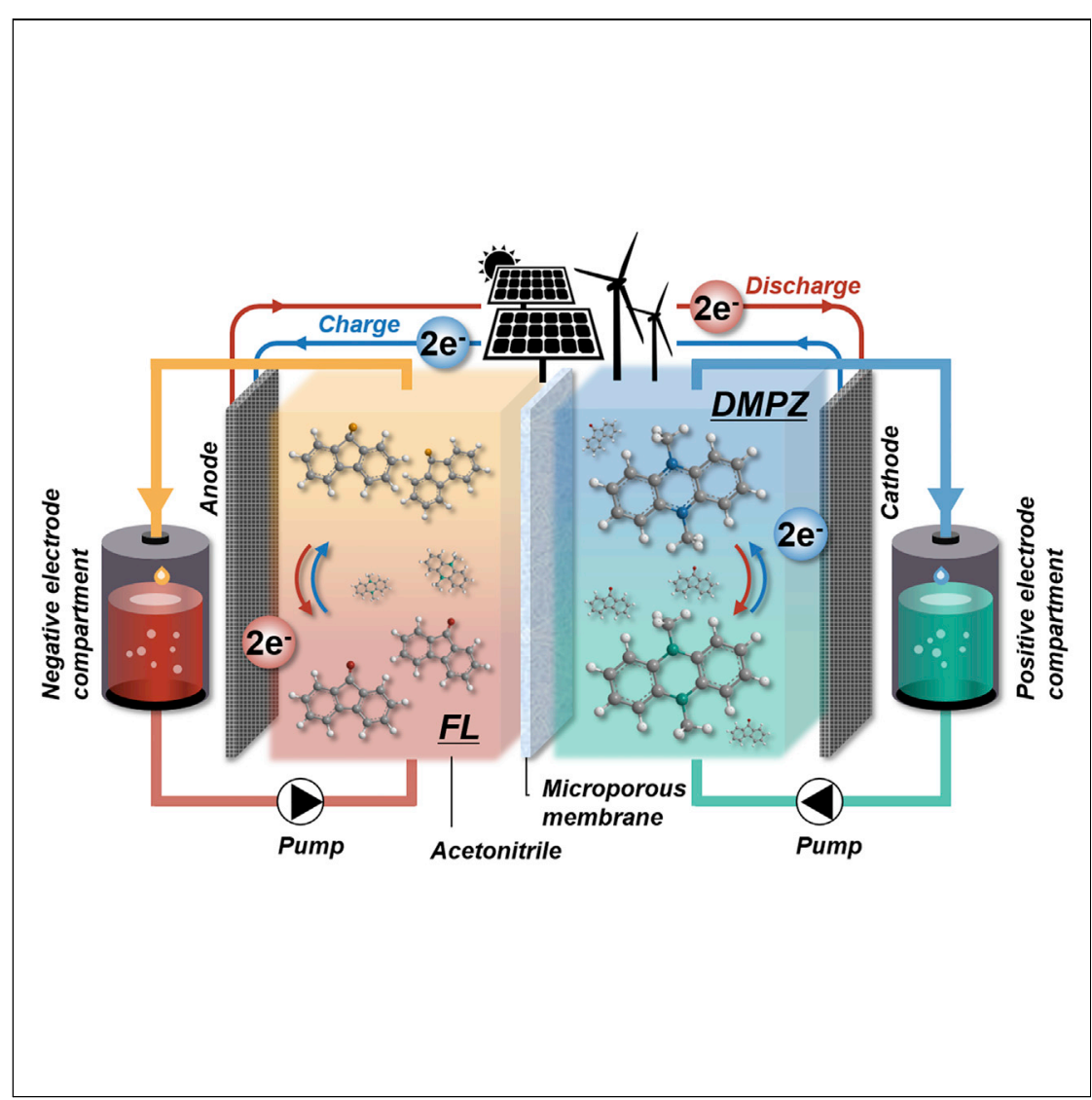

A multi-redox phenazine molecule $(5,10$-dihydro-5,10-dimethyl phenazine or DMPZ) is used as a positive electrode material for redox flow batteries to boost energy density. Its redox mechanism and chemical stability are investigated. The marked color change during redox reaction can be utilized for the estimation of the state of charge.
Giyun Kwon, Sechan Lee, Jinyeon Hwang, ..., Kyojin Ku, Jihyun Hong, Kisuk Kang

matlgen1@snu.ac.kr

HIGHLIGHTS

A multi-redox positive electrode material is developed for redox flow batteries

DMPZ exhibits double-redox reactions at high redox potentials

DMPZ/FL redox couple shows stable performance in flow cell systems

The estimation of the state of charge can be made using color change 


\title{
Article
}

\section{Multi-redox Molecule for High-Energy Redox Flow Batteries}

\author{
Giyun Kwon, ${ }^{1}$ Sechan Lee, ${ }^{1}$ Jinyeon Hwang, ${ }^{2}$ Hyun-Soo Shim, ${ }^{1}$ Byungju Lee, ${ }^{1}$ Myeong Hwan Lee, \\ Youngmin Ko, ${ }^{1}$ Sung-Kyun Jung, ${ }^{1}$ Kyojin Ku, ${ }^{1}$ Jihyun Hong, ${ }^{1}$ and Kisuk Kang ${ }^{1,3, *}$
}

\begin{abstract}
SUMMARY
Redox flow batteries (RFBs) are some of the most promising energy storage systems because of their design flexibility; however, their low energy density is a major drawback limiting widespread application. Most conventional approaches to increase the energy density have involved exploiting high-concentration electrolytes. However, this approach results in many technical issues, such as sluggish kinetics. We propose a strategy of boosting the energy density by exploiting a multi-redox phenazine molecule $(5,10$-dihydro-5,10-dimethyl phenazine [DMPZ]). DMPZ exhibits double-redox activity at -0.15 and $0.61 \mathrm{~V}$ versus $\mathrm{Ag} / \mathrm{Ag}^{+}$with remarkable kinetics and chemical stability. Coupled with 9-fluorenone (FL), the DMPZ/FL flow cell can provide the highest energy density per mole ( $\approx 85 \mathrm{~W} \mathrm{hr} \mathrm{mol}^{-1}$ ) ever reported for RFBs. Furthermore, the marked color change of DMPZ enables the state of charge to be precisely visualized. This novel strategy for a multi-redox material can provide a potential pathway toward high-energy-density RFBs.
\end{abstract}

\section{INTRODUCTION}

Concerns over fossil fuel depletion and greenhouse gas emissions have led to a high demand for sustainable energy resources such as solar and wind power in recent years; however, their full utilization in electric generation is partly limited by the intermittent nature of their supply. ${ }^{1,2}$ To overcome this issue, large-scale energy storage systems (ESSs) are needed, with rechargeable batteries being considered the most affordable choice. ${ }^{3}$ Among rechargeable batteries, redox flow batteries (RFBs) have received intense interest as one of the most attractive candidates for these applications because of their easy scale-up combined with cost-effectiveness and design flexibility that can decouple power and energy for diverse purposes. ${ }^{4-10}$ In particular, recent developments with non-aqueous systems employing redoxactive organic materials (ROMs) have provided a path toward the development of high-energy-density RFBs, while conventional aqueous RFBs have suffered from the low energy density arising from the narrow electrochemical voltage window limited by water electrolysis. ${ }^{7,11-13}$ Moreover, the potential low costs and chemical/structural diversity of ROMs offer improvements over those of transition-metalbased redox-active materials such as vanadium, chrome, or zinc, commonly used for conventional RFBs. ${ }^{14-16}$

Several important high-performance non-aqueous RFBs have recently been reported, such as systems based on 2,2,6,6-tetramethyl-1-piperidinyloxy (TEMPO) and 2,5-di-tert-butyl-1-methoxy-4-[2'-methoxyethoxy]benzene (DBMMB), which presented impressively higher energy density and cell voltage $(>1.6 \mathrm{~V})$ than transition-metal-based aqueous systems. ${ }^{17-23}$ In particular, DBMMB exhibited redox

\section{Context \& Scale}

Redox flow batteries (RFBs) are some of the most promising systems for large-scale energy storage because of their great scalability and design flexibility. Even though high-energy-density RFBs are required for practically feasible applications, critical bottlenecks still remain, such as increased electrolyte viscosity resulting from the use of highly concentrated electrolyte. Herein, we propose a novel strategy of boosting the energy density by utilizing a multi-redox phenazine molecule $(5,10$-dihydro-5,10dimethyl phenazine [DMPZ]). DMPZ can be used as a positive electrode material because of its double-redox reactions at high redox potentials with fast massand charge-transfer kinetics. Coupled with benchmarked negative electrode material (9-fluorenone [FL]), DMPZ/FL redox couple shows stable cell performance and the highest energy density among reported RFBs. These findings might shed light on the development of highenergy-density RFBs. 
activity at $0.73 \mathrm{~V}$ (versus $\mathrm{Ag} / \mathrm{Ag}^{+}$) in acetonitrile (MeCN) solvent-based electrolyte, delivering $\approx 60 \mathrm{~W} \mathrm{hr} \mathrm{mol}^{-1}$ when combined with 9-fluorenone $(\mathrm{FL})$, which is the highest value among non-aqueous organic RFBs reported to date. ${ }^{19}$ In attempts to achieve higher energy densities, increasing the concentration of these ROMs in the electrolyte has been a general and effective strategy to enable it to contain a larger amount of redox-active materials per system volume. ${ }^{17,21,24}$ For instance, Wei et al. ${ }^{17}$ demonstrated that the energy density of RFBs can be systematically improved by increasing the concentration of TEMPO up to $2.0 \mathrm{M}$. Milshtein et al. ${ }^{21}$ successfully showed that the energy density could be further enhanced by tailoring the solubility of $\mathrm{N}$-ethylphenothiazine in $\mathrm{MeCN}$ through chemical modification. Although this approach of exploiting a high-concentration electrolyte is simple and effective, there are unfavorable trade-offs, such as increased electrolyte viscosity and high cell resistance; in addition, it often leads to the precipitation of salts, which causes the failure of the system. ${ }^{17,20,25,26}$ Furthermore, non-aqueous RFBs can be more vulnerable to these trade-offs because of the intrinsically lower ionic conductivity of the non-aqueous electrolyte compared with its aqueous counterparts, which typically results in inferior power capability of the system. $20,27,28$

Herein, we propose that multi-electron ROMs can offer an alternative route for the preparation of high-energy-density RFBs. Unlike doubling the electrolyte concentration, for instance, which would easily result in the solubility limit being exceeded or significant reduction in the kinetics, the utilization of the double-redox activity from a single ROM would approximately double the energy density without negatively affecting the electrolyte properties. Nevertheless, the use of a second-electron redox reaction in most ROMs has often been shown to be irreversible or to promote severe degradation because of the poor chemical stability of the ROMs at highly oxidized states in the solution. ${ }^{20,21}$ Even though there are a few reports on candidates for multi-redox ROMs in recent years, they have not been demonstrated for full flow cell system. ${ }^{29,30}$ In this work, we introduce a novel multi-electron-redox phenazine molecule, 5,10-dihydro-5,10-dimethyl phenazine (DMPZ), as a new class of ROMs for the positive electrode compartment and demonstrate it for an allorganic flow system. Double-redox activities are observed, with the second-electron redox potential being one of the highest values reported for ROMs, and these reactions are remarkably reversible in conventional non-aqueous medium for RFBs with fast kinetics and chemical stability, thereby resulting in the highest energy density per mole among RFBs reported thus far.

\section{RESULTS AND DISCUSSION}

\section{A Multi-redox Molecule, DMPZ}

The phenazines are a group of biomolecules involved in the metabolism of living organisms, where various electron-transfer reactions occur, and are capable of performing redox reactions. ${ }^{31-37}$ DMPZ, a methylated phenazine at both nitrogen atoms in the pyrazine ring, is known to exhibit reversible two-step reactions of single-electron redox on the diazabutadiene motif in the heterocyclic system. ${ }^{38-40}$ Inspired by its electrochemical activity not only in aqueous biological systems but also in non-aqueous media with high redox potential and chemical stability, ${ }^{38-41}$ we explored here the double-redox activities of DMPZ for use as a positive electrode material in RFBs. The molecular structure of the redox DMPZ is presented in Figure $1 \mathrm{~A}$ along with the expected half-cell positive electrode reaction. The figure also shows the half-cell negative electrode reaction of $F L$, which we employed for preparing an all-organic RFB. ${ }^{19}$ Because of the expected multi-redox capability of DMPZ, a molar ratio of 1:2 DMPZ:FL was used in the construction of a flow cell

\author{
(1)
}

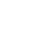


A

Positive electrode reaction<smiles>Cc1ccc2c(c1)N(C)c1ccccc1N2C</smiles>
DMPZ<smiles>C=Cc1ccc2c(c1)N(C)c1ccccc1N2C</smiles>

${ }_{D M P Z}{ }^{+}$<smiles>CN1c2ccccc2N(C)c2ccccc21</smiles>

$D_{M P Z}{ }^{2+}$

Negative electrode reaction<smiles>[Z7]c1ccc2c(c1)C(=O)c1ccccc1-2</smiles>

C

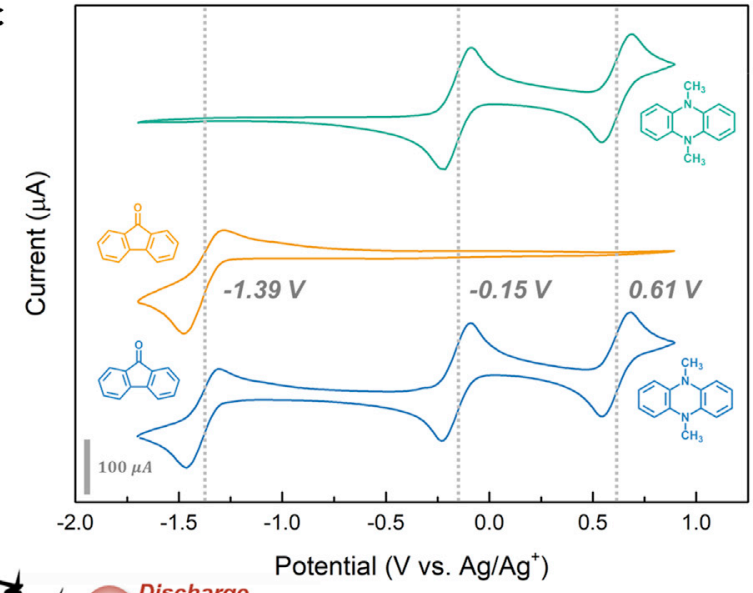

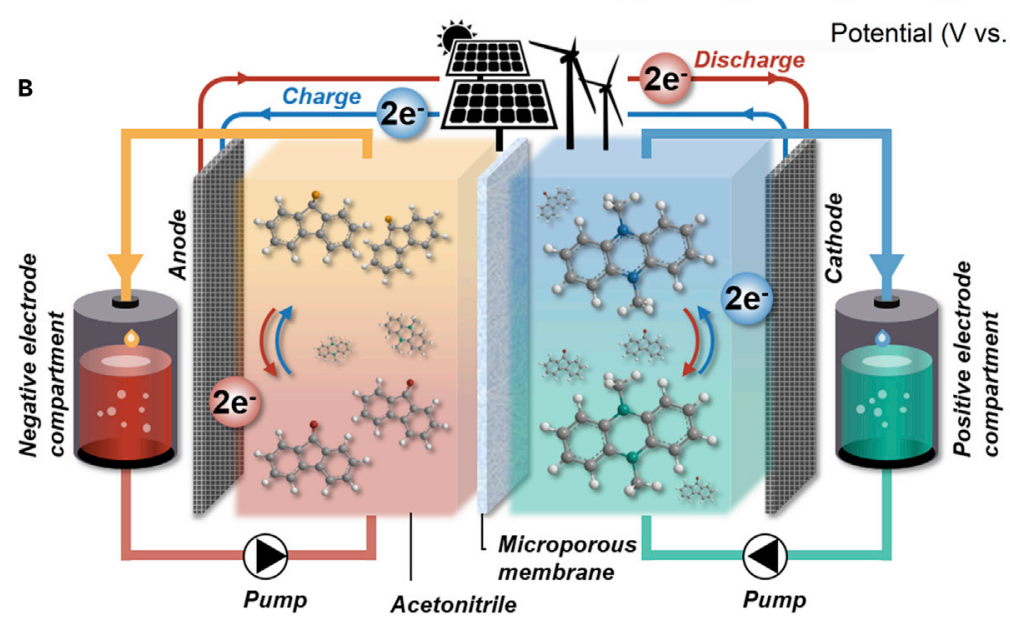

Figure 1. Electrochemical Reaction of DMPZ and FL

(A) Molecular structures and redox mechanisms of DMPZ and FL.

(B) Schematic illustration of energy storage in DMPZ/FL flow battery and cell configuration.

(C) CV curves of $10 \mathrm{mM} \mathrm{ROMs} \mathrm{in} \mathrm{supporting} \mathrm{electrolyte} \mathrm{of} 0.1 \mathrm{M} \mathrm{LiTFSI} \mathrm{in} \mathrm{MeCN} \mathrm{at} \mathrm{a} \mathrm{scan} \mathrm{rate} \mathrm{of} 100 \mathrm{mV} \mathrm{s}{ }^{-1}$ : DMPZ (green line), FL (orange line), mixture of FL and DMPZ (blue line).

comprising the DMPZ (positive electrode) and FL (negative electrode) using a supporting electrolyte of $\mathrm{MeCN},{ }^{19}$ which is schematically illustrated in Figure $1 \mathrm{~B}$. A microporous membrane was adopted for the mixed electrolyte, in which both DMPZ and FL were dissolved, to alleviate the crossover of the ROMs. ${ }^{19,42-44}$ It should be noted that the low-donor-number MeCN (14.1) was employed in utilizing the multi-electron redox reaction of DMPZ because the reversible second redox reaction of DMPZ was hindered in high-donor-number solvents such as DMSO (29.8) with the demethylation reaction. ${ }^{39}$ The electrochemical reaction of the cell begins with DMPZ first being oxidized upon charge (blue arrow), providing two electrons; $\mathrm{DMPZ}^{2+}$ is then reversibly reduced to DMPZ during discharge (red arrow).

Cyclic voltammograms ( $C V s$ ) were obtained for DMPZ and FL to investigate the redox activities and potentials of the redox couple in the $\mathrm{MeCN}$ electrolyte. As observed in Figure 1C, DMPZ (green line) displays two reversible redox peaks with average redox potentials of -0.15 and $0.61 \mathrm{~V}$ versus $\mathrm{Ag} / \mathrm{Ag}^{+}$, which are on a par with or higher than those of other reported ROMs, and FL (orange line) exhibits a reversible redox activity with an average potential of $-1.39 \mathrm{~V}$ (versus $\mathrm{Ag} / \mathrm{Ag}^{+}$), which is consistent with previous observations. ${ }^{18,21,45,46}$ It is noteworthy that the second redox potential of $0.61 \mathrm{~V}$ (versus $\mathrm{Ag} / \mathrm{Ag}^{+}$) for $\mathrm{DMPZ}$ is one of the highest values 

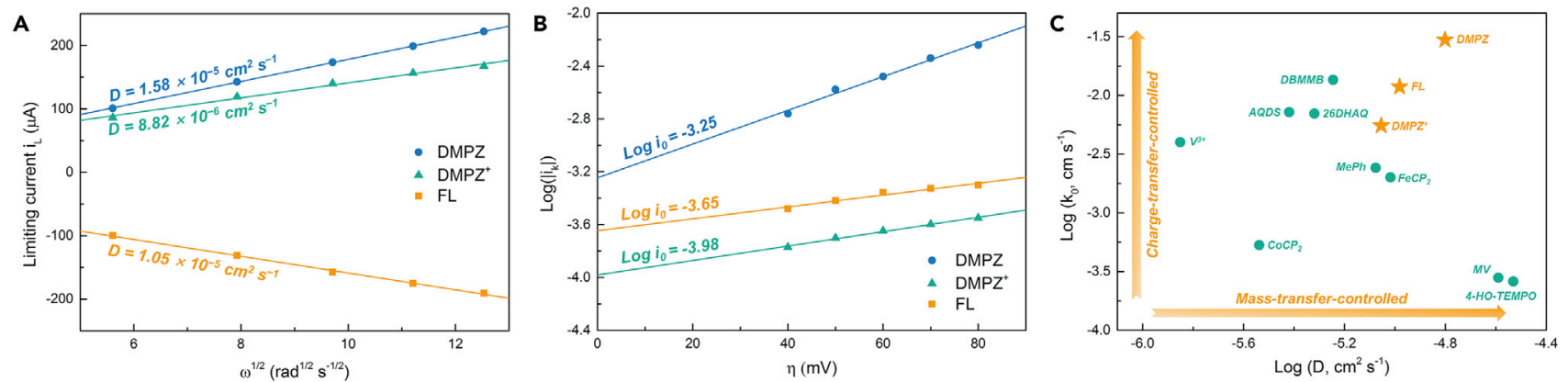

Figure 2. Electrochemical Kinetics of DMPZ, DMPZ+, and FL from RDE Studies with 1.0 mM ROMs in 0.5 M LiTFSI in MeCN

(A) Linearly fitted Levich plots of limiting current (iL) as a function of the square root of the rotation rates $\left(\omega^{-1 / 2}\right)$.

(B) Linearly fitted plots of logarithm of kinetics-controlled current (log $\left.i_{k}\right)$ versus overpotential $(\eta)$.

(C) Kinetic parameter comparison for typical redox-active materials in RFB system in terms of the kinetic rate constant $\mathrm{k}_{0}$ and diffusion coefficient $\mathrm{D}$ : this work (orange stars) and reported RFBs (green circles).

reported for ROMs to date. We obtained an additional CV for the mixture of DMPZ and FL to confirm the compatibility of these two redox species in the electrolyte during oxidation and reduction. The blue line in the CV of Figure $1 \mathrm{C}$ indicates that DMPZ and FL maintain the same redox potentials with currents equivalent to those of the individual cases, implying the absence of apparent side reactions among DMPZ/ $\mathrm{DMPZ}^{+} / \mathrm{DMPZ}^{2+}$ and $\mathrm{FL} / \mathrm{FL}^{-}$. Based on the $\mathrm{CVs}$, it is expected that a flow battery using a DMPZ/FL couple would harvest high theoretical cell voltages of 1.24 and $2 \mathrm{~V}$.

Investigation of the Electrochemical Kinetics of DMPZ/FL

The electrochemical kinetic properties of DMPZ in the electrolyte were examined using a series of rotating disk electrode (RDE) tests, as shown in Figure 2. As DMPZ undergoes multi-electron redox reactions, both the DMPZ and DMPZ ${ }^{+}$species were considered and treated as individual entities in the analysis. The linear sweep voltammetry (LSV) curves for DMPZ, DMPZ ${ }^{+}$, and FL reveal that the masstransport-controlled limiting currents $\left(i_{L}\right)$ increase with increasing rotation rates $(\omega)$ in the range of 300-1,500 rpm (Figures S1A-S1C). The mass-transfer-controlled kinetics were then analyzed using the Levich equation, which determines how fast the mass diffuses to the electrode in the Nernst diffusion layer. ${ }^{47}$ From the well-fitted Levich plots, which show the linear relationship between $i_{L}$ and the square root of $\omega$ (Figure $2 \mathrm{~A}$ ), the diffusion coefficients (D) of DMPZ, DMPZ ${ }^{+}$, and FL were calculated to be $1.58 \times 10^{-5}, 8.82 \times 10^{-6}$, and $1.05 \times 10^{-5} \mathrm{~cm}^{2} \mathrm{~s}^{-1}$, respectively (a detailed description of the calculations is provided in the Electrochemistry section of the Experimental Procedures). In addition, the charge-transfer-controlled kinetics, which contribute to the reaction rate of the electrode, were investigated using the Koutecky-Levich equation. ${ }^{47}$ The Koutecky-Levich plots at different overpotentials $(\eta)$ were extrapolated to determine the kinetic-controlled currents $\left(i_{k}\right)$ (Figures S1D-S1F). From the linearly fitted plots of $\log i_{k}$ versus the overpotentials in Figure $2 \mathrm{~B}$, the logarithms of the exchange currents ( $\log \mathrm{i}_{0}$ ) were determined to be -3.25 for $\mathrm{DMPZ},-3.98$ for $\mathrm{DMPZ}^{+}$, and -3.65 for $\mathrm{FL}$, yielding kinetic rate constants $\left(k_{0}\right)$ of $2.97 \times 10^{-2}, 5.53 \times 10^{-3}$, and $1.18 \times 10^{-2} \mathrm{~cm} \mathrm{~s}^{-1}$, respectively. It is worth noting that DMPZ has one of the highest kinetic rate constants of known ROMs. Figure $2 \mathrm{C}$ displays both the mass- and charge-transfer-controlled kinetic parameters of DMPZ, DMPZ ${ }^{+}$, and FL (orange stars) on a logarithm scale for comparison with previously reported redox-active materials in RFBs (green closed circles). Notably, the DMPZ/FL couple displayed outstanding kinetic characteristics in terms of both $D$ and $k_{0}$, exceeding those of other species, which implies that the observed fast kinetic 
A

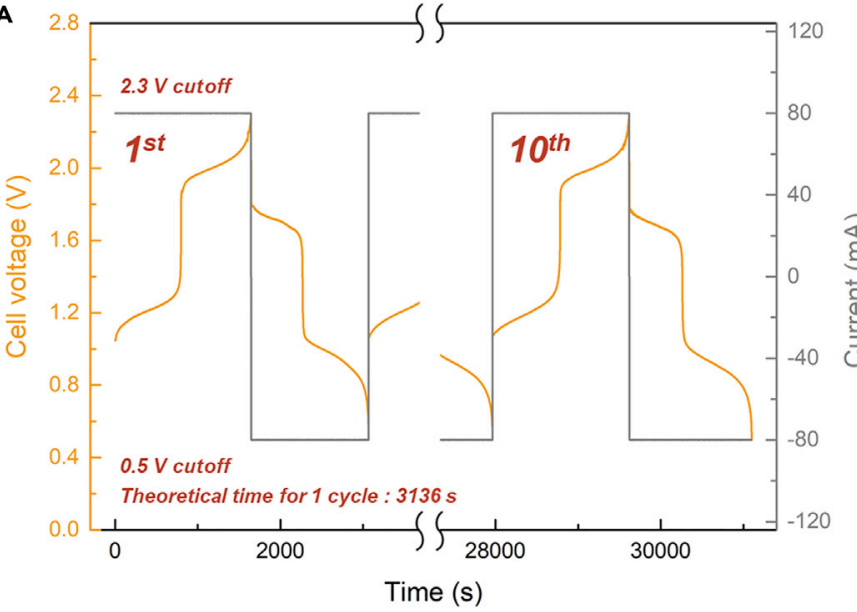

C

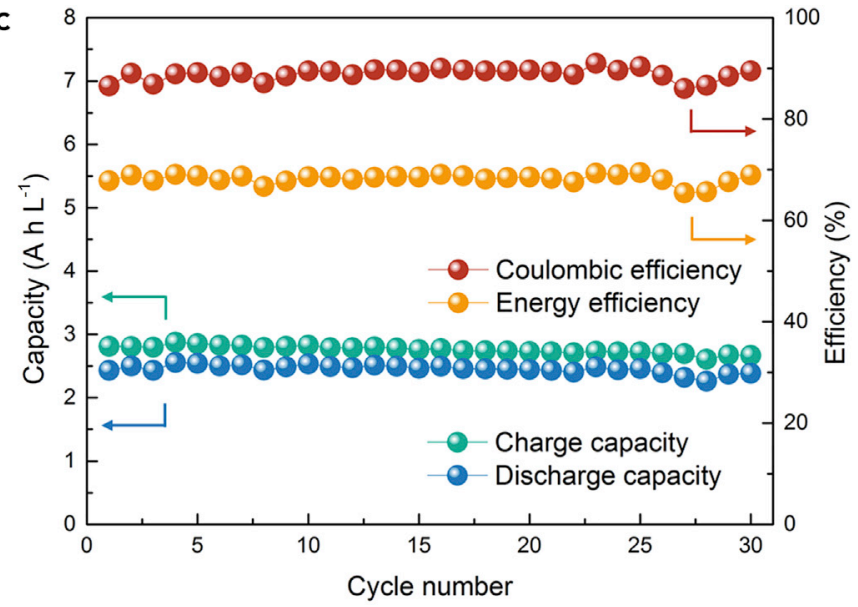

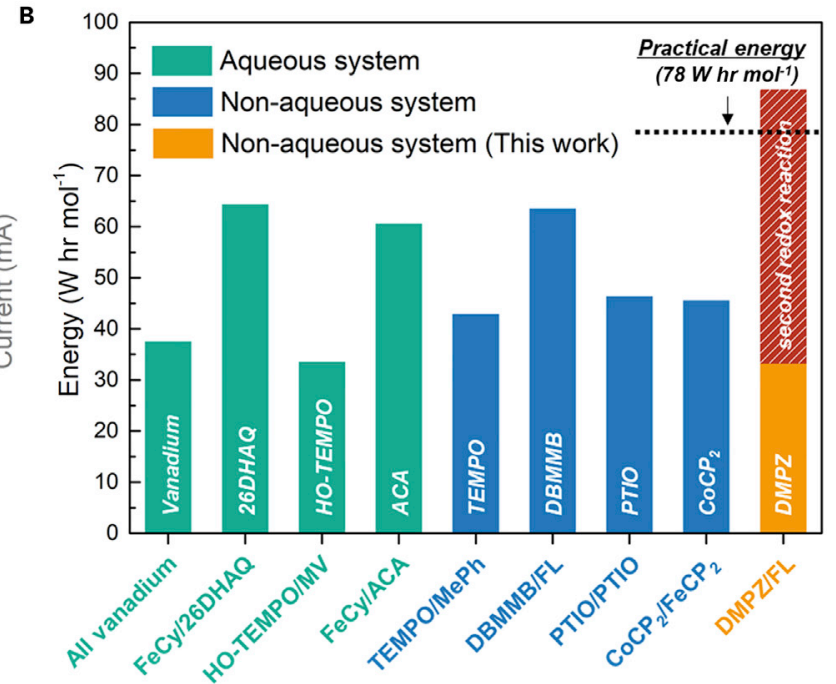

D

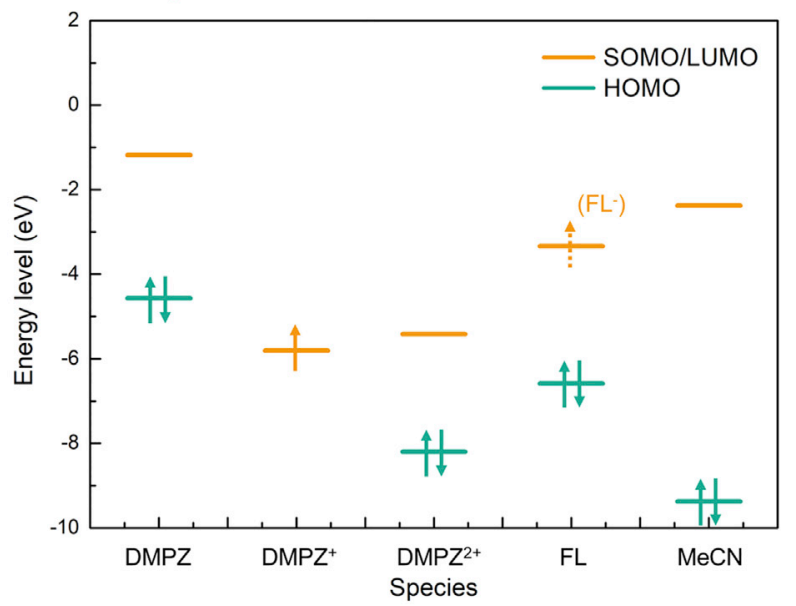

Figure 3. Electrochemical Performance of DMPZ/FL Flow Cell

(A) Cell voltage versus time for first and tenth cycles at $20 \mathrm{~mA} \mathrm{~cm}^{-2}$

(B) Energy comparison of typical redox-active materials in RFB; the dashed area denotes the contribution of the second redox reaction. Note that the energy density of the positive electrode compartment (energy density per volume) is determined by the intrinsic redox activity of the molecule (energy density per mole) and its solubility in the solvent (mole per volume). In order to emphasize the importance of the multi-redox reaction with respect to enhancing the intrinsic redox activity of molecules, we used the term "energy density per mole" here instead of "energy density per volume." Our intention is to decouple the engineering of the intrinsic redox activity and the solubility (see Figure S10 for details).

(C) Cycling efficiencies and capacities with respect to cycle number.

(D) Molecular orbital energies of ROMs and MeCN. Note that the energy states of DMPZ change with the oxidation due to the relaxation effect of the molecules.

parameters would have a positive effect on the cell polarization and energy efficiency, as illustrated later in detail.

\section{Cycling of the DMPZ/FL Flow Cell}

The electrochemical performance of flow cells employing a DMPZ positive electrode material and FL negative electrode material was investigated using a custom cell, the design of which is described in the Experimental Procedures. Figure 3A presents representative voltage versus time curves for the first and tenth cycles at $20 \mathrm{~mA} \mathrm{~cm}{ }^{-2}$ (Figure S2). Two well-defined plateaus were observed at 1.2 and $2 \mathrm{~V}$, which are consistent with the CV results in Figure 1C. Given the same lengths of the plateaus, it can be inferred that the two single-electron reactions contribute 
equally to the redox reaction and capacity. The Raman spectroscopy analysis in Figure S3 supports the view that the changes in the local bonding of DMPZ are reversible upon charging and discharging. The reversible bonding is associated with the vibrational modes of $\mathrm{C}-\mathrm{N}-\mathrm{C}$ and $\mathrm{C}=\mathrm{C}$ bonds around the diazabutadiene, in agreement with the reported redox mechanism, ${ }^{39}$ confirming the two-step multi-redox reactions in DMPZ during the flow cell operation (Table S1). To compare the performance of the DMPZ/FL flow cell with that of reported RFBs, the theoretical energy densities of RFBs employing both aqueous and nonaqueous media are plotted in Figure $3 \mathrm{~B}$ for the same concentration of ROMs. Figure 3B shows that DMPZ (versus FL) presents the highest theoretical and practical energy density among reported redox couples for both aqueous and non-aqueous RFBs, even exceeding those of recently reported RFBs based on DBMMB with the highest energy demonstrated thus far. ${ }^{19}$ It is worth noting that, for DMPZ, the energy density contributed from the second redox reaction is considerably larger than that contributed from the first redox reaction because of the higher second redox potential with the same redox capacity, which results in DMPZ outperforming the other materials with respect to the energy density.

Figure $3 C$ shows the energy efficiencies and capacity retention of the RFBs employing the DMPZ as a positive electrode material. The flow cell maintained a high coulombic efficiency (CE) of $\approx 90 \%$ and a voltage efficiency of $78 \%$ over 30 cycles, yielding a respectable energy efficiency of $\approx 70 \%$. Considering the high applied current density of $20 \mathrm{~mA} \mathrm{~cm}{ }^{-2}$, the observed efficiency indicates rapid kinetics in the electrochemical reaction, which outperforms other non-aqueous flow cells and is even comparable with those of some aqueous systems. ${ }^{15,18,19,24,48}$ The fast electrochemical reactions are attributed to the observed high kinetic rate constants $\left(k_{0}\right)$ and diffusion coefficients $(D)$ of the DMPZ and $F L$, as demonstrated in Figure 2. Stable cycling was also achieved for the flow cell without noticeable capacity fading, with retention of $\approx 98 \%$ of the initial capacity $\left(2.43 \mathrm{~A} \mathrm{hr} \mathrm{L}^{-1}\right)$ after 30 cycles and capacity retention of $\approx 90 \%$ was obtained after 80 cycles in the dilute condition (Figure S4). Note that the initial capacity of $2.43 \mathrm{~A} \mathrm{hr} \mathrm{L}^{-1}$ is close to the theoretical capacity of $2.68 \mathrm{~A} \mathrm{hr} \mathrm{L}^{-1}$, indicating $>90 \%$ utilization of the ROM. The stable cycle performance of the multi-redox flow cell is mainly attributed to the chemical stability and compatibility of the $\mathrm{DMPZ}^{2+}$ in the electrolyte, whereas most ROMs with the second oxidation tend to destabilize the system, leading to deterioration of the cycle performance. ${ }^{20,21,49}$ To comprehend the chemical stability of the redox couples in the given $\mathrm{MeCN}$-based electrolyte, the electronic structures of $\mathrm{DMPZ}, \mathrm{DMPZ}^{+}, \mathrm{DMPZ}^{2+}, \mathrm{FL}$, and $\mathrm{FL}^{-}$were compared with that of $\mathrm{MeCN}$ using density functional theory (DFT) calculations, as illustrated in Figure 3D. It was observed that the energy levels of the lowest unoccupied molecular orbital (LUMO) of DMPZ and the singly occupied molecular orbital (SOMO) of the radical $\mathrm{DMPZ}^{+}$were both higher than the highest occupied molecular orbital (HOMO) level of MeCN. More importantly, the LUMO level of $\mathrm{DMPZ}^{2+}$ was located above the $\mathrm{HOMO}$ level of $\mathrm{MeCN}$, indicating that the electron transfer from MeCN to DMPZ in any oxidation state during the battery operation is unfavorable, thus implying the electrochemical stability of DMPZ. Similarly, the electron transfer from DMPZ in any oxidation state to $\mathrm{MeCN}$ is energetically limited because the HOMO (or SOMO) levels of DMPZ and $\mathrm{DMPZ}^{2+}$ (or $\mathrm{DMPZ}^{+}$) are lower than the LUMO level of MeCN. Notably, although electron transfer from $\mathrm{FL}$ to $\mathrm{MeCN}$ or from $\mathrm{MeCN}$ to $F L$ is not likely because of their relative energy levels, similar to the case of DMPZ, there is a chance for the recombination between $\mathrm{DMPZ}^{+}$and $\mathrm{FL}^{-}$because of the higher SOMO level of $\mathrm{FL}^{-}$than that of $\mathrm{DMPZ}^{+}$, suggesting electron transfer from $\mathrm{FL}^{-}$to $\mathrm{DMPZ}^{+}$. Although this type of recombination is expected in mixed 
electrolyte systems, it is interesting to observe that $\mathrm{DMPZ}^{2+}$ can be more stable than $\mathrm{DMPZ}^{+}$against the recombination with $\mathrm{FL}^{-}$because of the fully occupied HOMO level and the LUMO level that is slightly higher than the SOMO level of $\mathrm{DMPZ}^{+}$. This finding may imply that the use of double-redox ROMs is not necessarily less stable against the self-discharge of RFBs exploiting mixed electrolyte systems despite the higher oxidation states of ROMs that may more strongly attract the counter ROMs. These theoretical calculations support the observed chemical and electrochemical stability of the MeCN-based flow cell with the double-redox DMPZ combined with FL. The time-dependent degradation of radical species as a form of $\mathrm{DMPZ}^{+}$is also investigated using UV-visible (UV-vis) spectroscopy because the stability of intermediate radicals is critically important in the RFB system utilizing ROMs. ${ }^{21,50,51}$ Interestingly, we observed the absorption spectra of the radical species is well maintained even after $24 \mathrm{hr}$, which implies DMPZ ${ }^{+}$has a superior radical stability (Figure S5).

\section{The Color Change of DMPZ for the Estimation of the State of Charge}

One of the interesting properties of the DMPZ/FL flow battery is that the state of charge (SOC) can be visually and precisely estimated by probing the color change of DMPZ. Close monitoring of the SOC is critical in a battery system to prevent potential safety issues such as gas evolution. ${ }^{52}$ Moreover, for new large-scale applications such as electric vehicles and ESSs, this monitoring becomes more vital because it informs the user when to charge and prevents the overcharging of batteries. Although electron spin resonance spectroscopy has been used to determine the $\mathrm{SOC}$ in RFBs, ${ }^{17}$ it is not practically viable, and, for multi-electron ROMs, there is not a one-to-one correspondence between the radical concentration of these ROMs and the SOC. Taking advantage of the marked color change of DMPZ from yellow to green, orange, and red at different charged states, simple and rapid prediction of the SOC was possible. In the quantitative analysis, UV-vis spectroscopy enabled accurate measurement of the SOC based on Beer's law. ${ }^{53}$ Figure 4 presents the UV-vis spectra and color changes of DMPZ in the positive electrode compartment as a function of the SOC (with a step size of SOC 25). Color changes were observed during two stages in the charging process and were attributed to the double-redox reaction of DMPZ. In the first stage (SOC 0-50), during which the $\mathrm{DMPZ}^{+}$concentration increased, the yellow color became green, and in the UV-vis spectra (left in the figure) the intensities of the three peaks in the range of 580-750 nm gradually increased. In the second stage (SOC 50 to 100), during which $\mathrm{DMPZ}^{+}$was oxidized to $\mathrm{DMPZ}^{2+}$, the dark green color became orange and finally red. In this stage, the peak intensities in the range of 580-750 nm decreased, and new peaks appeared in the range of 500-560 nm. Reversible changes of the color and UV-vis spectra were also observed upon discharging, providing further confirmation of these changes. For the quantitative determination of the SOCs, we selected two representative peaks at 660 and $521 \mathrm{~nm}$ in the UV-vis spectra for the reference, which were correlated to the concentrations of $D M P Z^{+}$and $\mathrm{DMPZ}^{2+}$ depending on the SOCs. Linear relationships between the absorbance and SOCs were clearly observed for both peaks in each stage (i.e., the first and second redox reactions), confirming that accurate estimation of the SOCs is possible in a DMPZ/FL flow battery (Figure S6).

\section{Conclusion}

In summary, we employed, for the first time, a multi-electron redox DMPZ as a promising positive electrode material for high-energy-density RFBs. This novel ROM exhibited two reversible redox reactions at high redox potentials of -0.15 and $0.61 \mathrm{~V}$ versus $\mathrm{Ag} / \mathrm{Ag}^{+}$and presented outstanding electrochemical kinetic properties 


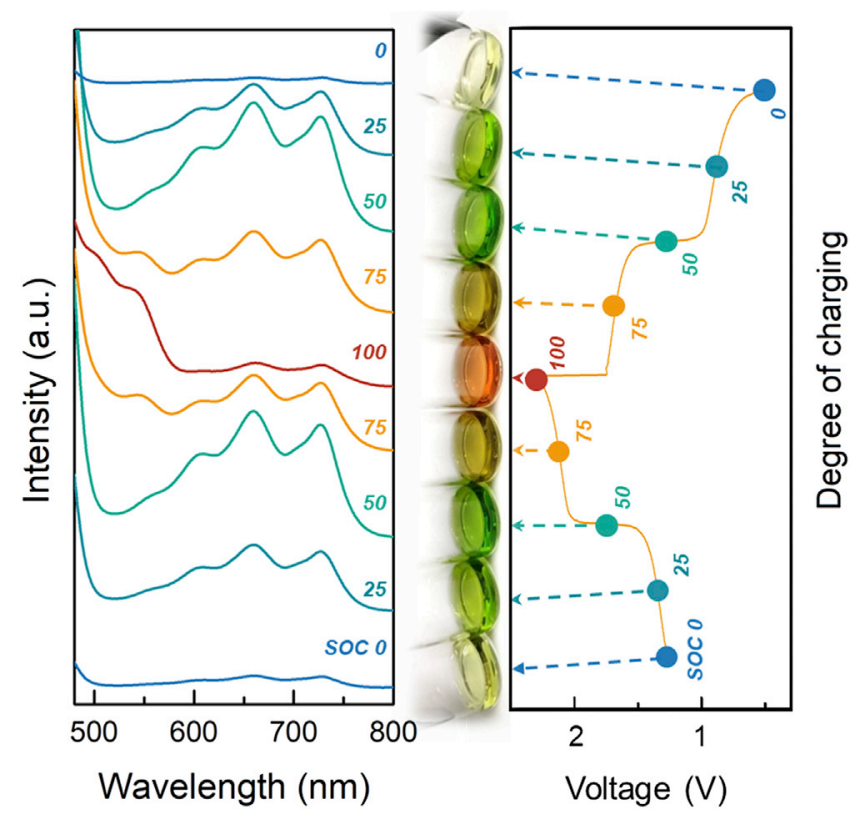

Figure 4. Prediction of SOC

Analysis of color changes of DMPZ as a function of the SOC using UV-vis spectroscopy; the dotted arrows point to the corresponding colors of the electrolytes in the positive electrode compartment. Four pieces of Celgard 4,560 were used to minimize the crossover and accurately measure the SOC, resulting in CE of $>99 \%$.

for both the mass- and charge-transfer reactions. The DMPZ/FL flow cell exhibited two well-defined voltage plateaus at 1.2 and $2 \mathrm{~V}$ with superior material utilization (>90\%) and remarkable chemical stability, delivering the highest energy per mole among reported aqueous and non-aqueous RFBs. In addition, the quantitative estimation of the SOC was possible based on the distinct color change of DMPZ in two stages, offering an additional advantage of the system. While the solubility of DMPZ $(\approx 60 \mathrm{mM})$ is not sufficient for direct application to ESSs, future efforts will aim to utilize the highly soluble version of DMPZ through the chemical modification; especially, we will focus on tailoring the molecular structure of DMPZ such as by attaching methoxy or ethoxy group to DMPZ to improve the solubility in MeCN-based solvents. ${ }^{21}$ These findings on this multi-electron redox material provide a potential new pathway for the design of high-energy-density RFBs and practically feasible ESSs.

\section{EXPERIMENTAL PROCEDURES}

Preparation of Materials

DMPZ was purchased from TCl Chemicals (Japan). Bis(trifluoromethane)sulfonimide lithium salt (LiTFSI), MeCN (anhydrous), and FL were purchased from Sigma-Aldrich and used without further purification. The microporous separator (Celgard 4560) was purchased from Wellcos (Korea) and treated under vacuum at $70^{\circ} \mathrm{C}$ for $24 \mathrm{hr}$ to remove moisture.

\section{Electrochemistry}

The solutions were prepared and the electrochemical tests were performed in an Arfilled glove box under an inert atmosphere (<0.5 ppm $\left.\mathrm{O}_{2}, \mathrm{H}_{2} \mathrm{O}\right)$. CVs of FL, DMPZ, and the mixture of FL and DMPZ (10 mM each) were obtained using a supporting electrolyte of $0.1 \mathrm{M} \mathrm{LiTFSI}$ in MeCN. A three-electrode system (Pt counter electrode, 
$\mathrm{Ag} / \mathrm{AgNO}_{3}$ reference electrode, Au working electrode) was employed with a scan rate of $100 \mathrm{mV} \mathrm{s}^{-1}$.

In the RDE test, a three-electrode system (Pt counter electrode, $\mathrm{Ag} / \mathrm{AgNo}_{3}$ reference electrode, glassy carbon working electrode with 5-mm diameter) was employed for the LSV tests. The DMPZ ${ }^{+}$solution was prepared from the DMPZ solution under constant-voltage mode at $0.2 \mathrm{~V}$ versus $\mathrm{Ag} / \mathrm{Ag}^{+}$. The working electrode was rotated from 300 to $1,500 \mathrm{rpm}$ (in increments of $300 \mathrm{rpm}$ ) using a modulated speed rotator (AFMSRX; PINE). The LSV tests were conducted with $1.0 \mathrm{mM} \mathrm{ROMs} \mathrm{in} 0.5 \mathrm{M}$ LiTFSI in $\mathrm{MeCN}$ at a scan rate of $5 \mathrm{mV} \mathrm{s}^{-1}$. The kinematic viscosity $(v)$ of the $0.5 \mathrm{M} \mathrm{LiTFSI}$ in $\mathrm{MeCN}$ was measured to be $0.59 \mathrm{~mm}^{2} \mathrm{~s}^{-1}$ following ASTM D445 in Koptri (Korea). Using the slopes of the linearly fitted Levich plots (Figure 2A) and the Levich equation (Equation 1), the diffusion coefficients (D) of the ROMs were calculated (the slopes of DMPZ, DMPZ ${ }^{+}$, and FL were determined to be $1.74 \times 10^{-5}, 1.18 \times 10^{-5}$, and $-1.32 \times 10^{-5} \mathrm{~A} \mathrm{rad}^{-1 / 2} \mathrm{~s}^{1 / 2}$, respectively).

$$
i_{L}=0.62 \mathrm{nFAD} D^{2 / 3} \omega^{1 / 2} v^{-1 / 6} C_{0} \quad \text { (Equation 1) }
$$

Here, $n$ is the number of electrons transferred $(n=1), F$ is the Faradaic constant $(F=$ $\left.96,485 \mathrm{C} \mathrm{mol}^{-1}\right), A$ is the electrode area $\left(A=0.2 \mathrm{~cm}^{2}\right)$, and $C_{0}$ is the concentration of $\operatorname{ROMs}\left(C_{0}=1.0 \mathrm{mM}\right)$.

A custom flow cell with backing plates (polyethylene-coated fiber glass), flow fields (polytetrafluoroethylene [PTFE]), and gaskets (PTFE) was fabricated using materials purchased from ILDO F\&C (Korea). The flow cells were assembled with carbon felts (XF30A; TOYOBO, Korea) as electrodes at both the anode and cathode side with two pieces of microporous separators (Celgard 4560) sandwiched in between. The active size of the flow cell was $2.0 \mathrm{~cm}$ wide $\times 2.0 \mathrm{~cm}$ long $\left(4 \mathrm{~cm}^{2}\right)$, and norprene tubing (Masterflex) was used. The electrolytes $(13 \mathrm{~mL}$ on each side) containing $\mathrm{FL}$ $(0.1 \mathrm{M})$ and DMPZ $(50 \mathrm{mM})$ in the supporting electrolyte of $0.5 \mathrm{M} \mathrm{LiTFSI}$ in MeCN

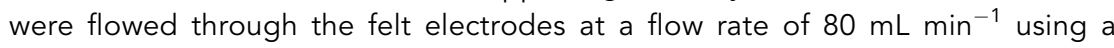
pump (ShenChen). The uniform volumetric porosity and the height of the carbon felts are $95 \%$ and $4.3 \mathrm{~mm}$, and accordingly the mean linear flow velocity of electrolyte past the electrode surface is calculated to be $1.63 \mathrm{~cm} \mathrm{~s}^{-1} \cdot{ }^{54,55}$ Flow cell tests were performed at room temperature in constant-current mode using a battery test system (WBCS 3000; WonA Tech, Korea). The volumetric energy density was calculated based on the electrolyte volume of the positive compartment $(13 \mathrm{~mL})$.

\section{Computational Details}

The Gaussian 09 quantum chemistry package ${ }^{56}$ was used for spin-unrestricted DFTtype calculations, which included geometry optimization, energy evaluation, eigenvalue calculation, and vibration mode prediction of the molecules.

The Becke-Lee-Yang-Parr (B3LYP) hybrid exchange-correlation functional ${ }^{57-59}$ and triple-zeta valence polarization basis $\operatorname{set}^{60,61}$ were used for the entire calculation. The polarizable continuum model scheme, which is embedded in Gaussian 09, was applied to implicitly consider the solvation of acetonitrile (dielectric constant $=38.8$.

The LUMO denotes the adiabatic LUMO.

\section{Characterization}

To prevent air contamination, all the samples were prepared and sealed in an Arfilled glove box. The electrolytes containing FL (20 mM) and DMPZ (10 mM) in the 
supporting electrolyte of 0.5 M LiTFSI in MeCN were used. For the UV-vis spectroscopy analysis, absorption spectra of the electrolytes in the positive electrode compartment (diluted in $\mathrm{MeCN}, 5 \% \mathrm{v} / \mathrm{v}$ ) were obtained using a UV-vis spectrometer (Agilent Technologies, Cary 5000) with an optical glass cuvette (Quartz; Hellma).

Raman spectra (LabRAM HR Evolution, Horiba) were recorded using capillary tubes (inner diameter of 1.1-1.2 mm) with continuous-wave linearly polarized lasers (wavelengths: 532, $633 \mathrm{~nm}$ ). The laser beam was focused using a $100 \times$ objective lens, resulting in a spot diameter of approximately $1 \mu \mathrm{m}$. The acquisition time and number of accumulations were $10 \mathrm{~s}$ and five, respectively.

\section{SUPPLEMENTAL INFORMATION}

Supplemental Information includes 10 figures, 1 table, and 1 note and can be found with this article online at https://doi.org/10.1016/j.joule.2018.05.014.

\section{ACKNOWLEDGMENTS}

This work was supported by (1) the National Research Foundation of Korea (NRF) grant funded by the Korean government (MSIP) (no. 2015R1A2A1A10055991), (2) Lotte Chemical, and (3) Creative Materials Discovery Program through the National Research Foundation of Korea (NRF) funded by the Ministry of Science, ICT and Future Planning (NRF-2017M3D1A1039553).

\section{AUTHOR CONTRIBUTIONS}

K. Kang conceived the original idea. G.K. designed the experiments with help from S.L., J. Hwang, H.-S.S., M.H.L., Y.K., S.-K.J., K. Ku, and J. Hong. The computations were performed by B.L. K. Kang supervised the overall research. All authors discussed the experiments and contributed to the final manuscript.

\section{DECLARATION OF INTERESTS}

The authors declare no competing interests.

Received: March 12, 2018

Revised: April 30, 2018

Accepted: May 25, 2018

Published: June 18, 2018

\section{REFERENCES}

1. Dunn, B., Kamath, H., and Tarascon, J.-M (2011). Electrical energy storage for the grid: a battery of choices. Science 334, 928-935.

2. Yang, Z., Zhang, J., Kintner-Meyer, M.C., Lu, X., Choi, D., Lemmon, J.P., and Liu, J. (2011). Electrochemical energy storage for green grid. Chem. Rev. 111, 3577-3613.

3. Zhao, H., Wu, Q., Hu, S., Xu, H., and Rasmussen, C.N. (2015). Review of energy storage system for wind power integration support. Appl. Energy 137, 545-553.

4. Nguyen, T., and Savinell, R.F. (2010). Flow batteries. Electrochem. Soc. Interface 19, 54-56.

5. Soloveichik, G.L. (2015). Flow batteries: current status and trends. Chem. Rev. 115, 1153311558.
6. Darling, R.M., Gallagher, K.G., Kowalski, J.A., $\mathrm{Ha}, \mathrm{S}$., and Brushett, F.R. (2014). Pathways to low-cost electrochemical energy storage: a comparison of aqueous and nonaqueous flow batteries. Energy Environ. Sci. 7, 3459-3477.

7. Wang, W., Luo, Q., Li, B., Wei, X., Li, L., and Yang, Z. (2013). Recent progress in redox flow battery research and development. Adv. Funct. Mater. 23, 970-986.

8. Leung, P., Li, X., De León, C.P., Berlouis, L., Low, C.J., and Walsh, F.C. (2012). Progress in redox flow batteries, remaining challenges and their applications in energy storage. RSC Adv. 2, 10125-10156.

9. De Leon, C.P., Frías-Ferrer, A., GonzálezGarcía, J., Szánto, D., and Walsh, F.C. (2006).

Redox flow cells for energy conversion. J. Power Sources 160, 716-732. 
14. Brushett, F.R., Vaughey, J.T., and Jansen, A.N. (2012). An all-organic non-aqueous lithium-ion redox flow battery. Adv. Energy Mater. 2, 13901396.

15. Song, Z., and Zhou, H. (2013). Towards sustainable and versatile energy storage devices: an overview of organic electrode materials. Energy Environ. Sci. 6, 2280-2301.

16. Jia, C., Pan, F., Zhu, Y.G., Huang, Q., Lu, L., and Wang, Q. (2015). High-energy density nonaqueous all redox flow lithium battery enabled with a polymeric membrane. Sci. Adv. 1, e1500886.

17. Wei, X., Xu, W., Vijayakumar, M., Cosimbescu, L., Liu, T., Sprenkle, V., and Wang, W. (2014). TEMPO-based catholyte for high-energy density nonaqueous redox flow batteries. Adv. Mater. 26, 7649-7653.

18. Li, Z., Li, S., Liu, S., Huang, K., Fang, D., Wang, F., and Peng, S. (2011). Electrochemical properties of an all-organic redox flow battery using 2, 2, 6, 6-tetramethyl-1-piperidinyloxy and $\mathrm{N}$-methylphthalimide. Electrochem. Solid State Lett. 14, A171-A173.

19. Wei, X., Xu, W., Huang, J., Zhang, L., Walter, E., Lawrence, C., Vijayakumar, M., Henderson, W.A., Liu, T., and Cosimbescu, L. (2015). Radical compatibility with nonaqueous electrolytes and its impact on an all-organic redox flow battery. Angew. Chem. Int. Ed. 54, 8684-8687.

20. Wei, X., Duan, W., Huang, J., Zhang, L., Li, B., Reed, D., Xu, W., Sprenkle, V., and Wang, W. (2016). A high-current, stable nonaqueous organic redox flow battery. ACS Energy Lett. 1, 705-711.

21. Milshtein, J.D., Kaur, A.P., Casselman, M.D. Kowalski, J.A., Modekrutti, S., Zhang, P.L. Attanayake, N.H., Elliott, C.F., Parkin, S.R., and Risko, C. (2016). High current density, long duration cycling of soluble organic active species for non-aqueous redox flow batteries. Energy Environ. Sci. 9, 3531-3543.

22. Milshtein, J.D., Barton, J.L., Darling, R.M., and Brushett, F.R. (2016). 4-acetamido-2, 2, 6, 6tetramethylpiperidine-1-oxyl as a model organic redox active compound for nonaqueous flow batteries. J. Power Sources $327,151-159$

23. Park, S.-K., Shim, J., Yang, J., Shin, K.-H., Jin, C.-S., Lee, B.S., Lee, Y.-S., and Jeon, J.-D (2015). Electrochemical properties of a nonaqueous redox battery with all-organic redox couples. Electrochem. Commun. 59, 68-71.

24. Takechi, K., Kato, Y., and Hase, Y. (2015). A highly concentrated catholyte based on a solvate ionic liquid for rechargeable flow batteries. Adv. Mater. 27, 2501-2506.

25. Duan, W., Huang, J., Kowalski, J.A., Shkrob, I.A., Vijayakumar, M., Walter, E., Pan, B., Yang, Z., Milshtein, J.D., and Li, B. (2017). "Wine-Dark Sea" in an organic flow battery: storing negative charge in 2,1,3-benzothiadiazole radicals leads to improved cyclability. ACS Energy Lett. 2,1156-1161.

26. Kosswattaarachchi, A.M., and Cook, T.R. (2018). Concentration-dependent charge discharge characteristics of non-aqueous redox flow battery electrolyte combinations. Electrochim. Acta 261, 296-306.
27. Winsberg, J., Janoschka, T., Morgenstern, S., Hagemann, T., Muench, S., Hauffman, G. Gohy, J.F., Hager, M.D., and Schubert, U.S (2016). Poly (TEMPO)/zinc hybrid-flow battery: a novel, "green," high voltage, and safe energy storage system. Adv. Mater. 28, 2238-2243.

28. Winsberg, J., Hagemann, T., Janoschka, T., Hager, M.D., and Schubert, U.S. (2016). Redoxflow batteries: from metals to organic redoxactive materials. Angew. Chem. Int. Ed. 56, 686-711.

29. Sevov, C.S., Hickey, D.P., Cook, M.E. Robinson, S.G., Barnett, S., Minteer, S.D., Sigman, M.S., and Sanford, M.S. (2017). Physical organic approach to persistent cyclable, low-potential electrolytes for flow battery applications. J. Am. Chem. Soc. 139, 2924-2927.

30. Kim, H.-S., Lee, K.-J., Han, Y.-K., Ryu, J.H., and Oh, S.M. (2017). A comparative study on the solubility and stability of p-phenylenediamine based organic redox couples for non-aqueous flow batteries. J. Power Sources 348, 264-269.

31. Rabaey, K., Boon, N., Höfte, M., and Verstraete, W. (2005). Microbial phenazine production enhances electron transfer in biofuel cells. Environ. Sci. Technol. 39, 3401-3408.

32. Wang, Y., Kern, S.E., and Newman, D.K. (2010) Endogenous phenazine antibiotics promote anaerobic survival of Pseudomonas aeruginosa via extracellular electron transfer. J. Bacteriol. 192, 365-369

33. Mavrodi, D.V., Blankenfeldt, W., and Thomashow, L.S. (2006). Phenazine compounds in fluorescent Pseudomonas spp. biosynthesis and regulation. Annu. Rev. Phytopathol. 44, 417-445.

34. Laursen, J.B., and Nielsen, J. (2004). Phenazine natural products: biosynthesis, synthetic analogues, and biological activity. Chem. Rev. 104, 1663-1686.

35. Chen, J.-J., Chen, W., He, H., Li, D.-B., Li, W.-W., Xiong, L., and Yu, H.-O. (2012) Manipulation of microbial extracellular electron transfer by changing molecular structure of phenazine-type redox mediators. Environ. Sci. Technol. 47, 1033-1039.

36. White, J.R., and Dearman, H.H. (1965). Generation of free radicals from phenazine methosulfate, streptonigrin, and riboflavin in bacterial suspensions. Proc. Natl. Acad. Sci. USA 54, 887-891.

37. Lee, B., Ko, Y., Kwon, G., Lee, S., Ku, K., Kim, J., and Kang, K. (2017). Exploiting biological systems: toward eco-friendly and highefficiency rechargeable batteries. Joule 2, $61-75$.

38. Lim, H.-D., Lee, B., Zheng, Y., Hong, J., Kim, J., Gwon, H., Ko, Y., Lee, M., Cho, K., and Kang, K. (2016). Rational design of redox mediators for advanced Li-O2 batteries. Nat. Energy 1, 16066.

39. Lee, M., Hong, J., Lee, B., Ku, K., Lee, S., Park, C.B., and Kang, K. (2017). Multi-electron redox phenazine for ready-to-charge organic batteries. Green Chem. 19, 2980-2985.

40. Nelson, R., Leedy, D., Seo, E., and Adams, R.N. (1966). Anodic oxidation of 5, 10-dihydro-5, 10-dimethylphenazine. Fresenius Z. Anal. Chem. 224, 184-196.

41. Lee, S., Kwon, G., Ku, K., Yoon, K., Jung, S.K., Lim, H.D., and Kang, K. (2018). Recent progress in organic electrodes for $\mathrm{Li}$ and $\mathrm{Na}$ rechargeable batteries. Adv. Mater. e1704682, https://doi.org/10.1002/adma.201704682.

42. Zhang, $H$., Zhang, $H ., L i, X$. Mai, Z., and Zhang, J. (2011). Nanofiltration (NF) membranes: the next generation separators for all vanadium redox flow batteries (VRBs)? Energy Environ. Sci. 4, 1676-1679.

43. Wei, X., Li, L., Luo, O., Nie, Z., Wang, W. Li, B., Xia, G.-G., Miller, E., Chambers, J., and Yang, Z. (2012). Microporous separators for $\mathrm{Fe} / \mathrm{V}$ redox flow batteries. J. Power Sources 218, 39-45.

44. Lopez-Atalaya, M., Codina, G., Perez, J., Vazquez, J., and Aldaz, A. (1992). Optimization studies on a Fe/Cr redox flow battery. J. Power Sources 39, 147-154.

45. Wang, W., Xu, W., Cosimbescu, L., Choi, D., Li, L., and Yang, Z. (2012). Anthraquinone with tailored structure for a nonaqueous metalorganic redox flow battery. Chem. Commun. (Camb.) 48, 6669-6671.

46. Duan, W., Vemuri, R.S., Milshtein, J.D. Laramie, S., Dmello, R.D., Huang, J., Zhang, L., Hu, D., Vijayakumar, M., and Wang, W. (2016). A symmetric organic-based nonaqueous redox flow battery and its state of charge diagnostics by FTIR. J. Mater. Chem. A 4 , 5448-5456.

47. Bard, A.J., and Faulkner, L.R. (2002). Fundamentals and Applications (Wiley).

48. Saraidaridis, J.D., Bartlett, B.M., and Monroe, C.W. (2016). Spectroelectrochemistry of vanadium acetylacetonate and chromium acetylacetonate for symmetric nonaqueous flow batteries. J. Electrochem. Soc. 163, A1239-A1246.

49. Huang, J., Pan, B., Duan, W., Wei, X., Assary, R.S., Su, L., Brushett, F.R., Cheng, L., Liao, C., and Ferrandon, M.S. (2016). The lightest organic radical cation for charge storage in redox flow batteries. Sci. Rep. 6, 32102 .

50. Beh, E.S., De Porcellinis, D., Gracia, R.L Xia, K.T., Gordon, R.G., and Aziz, M.J. (2017). A neutral pH aqueous organicorganometallic redox flow battery with extremely high capacity retention. ACS Energy Lett. 2, 639-644.

51. Chen, H., Cong, G., and Lu, Y.-C. (2018). Recent progress in organic redox flow batteries: active materials, electrolytes and membranes. J. Energy Chem. https://doi.org/10.1002/ adma.201704682.

52. Ding, C., Zhang, H., Li, X., Liu, T., and Xing, F. (2013). Vanadium flow battery for energy storage: prospects and challenges. J. Phys. Chem. Lett. 4, 1281-1294.

53. Cannon, C., and Butterworth, I. (1953). Beer's law and spectrophotometer linearity. Anal. Chem. 25, 168-170.

54. Park, S.-K., Shim, J., Yang, J.H., Jin, C.-S., Lee B.S., Lee, Y.-S., Shin, K.-H., and Jeon, J.-D. (2014). The influence of compressed carbon felt 
electrodes on the performance of a vanadium redox flow battery. Electrochim. Acta 116 447-452.

55. Arenas, L.F., de León, C.P., and Walsh, F.C. (2016). Mass transport and active area of porous Pt/Ti electrodes for the $\mathrm{Zn}$-Ce redox flow battery determined from limiting current measurements. Electrochim. Acta 221, 154-166.

56. Frisch, M., Trucks, G., Schlegel, H., Scuseria, G., Robb, M., Cheeseman, J., Scalmani, G.
Barone, V., Mennucci, B., and Petersson, G. (2016). Gaussian 09 (Gaussian).

57. Lee, C., Yang, W., and Parr, R.G. (1988) Development of the Colle-Salvetti correlationenergy formula into a functional of the electron density. Phys. Rev. B 37, 785.

58. Becke, A.D. (1993). Density-functional thermochemistry. III. The role of exact exchange. J. Chem. Phys. 98, 5648-5652.

59. Stephens, P., Devlin, F., Chabalowski, C., and Frisch, M.J. (1994). Ab initio calculation of vibrational absorption and circular dichroism spectra using density functional force fields. J. Phys. Chem. 98, 11623-11627.

60. Schäfer, A., Huber, C., and Ahlrichs, R. (1994). Fully optimized contracted Gaussian basis sets of triple zeta valence quality for atoms Li to $\mathrm{Kr}$. J. Chem. Phys. 100, 5829-5835.

61. Klaumünzer, B., Krüner, D., and Saalfrank, P. (2010). (TD-) DFT calculation of vibrational and vibronic spectra of riboflavin in solution. J. Phys. Chem. B 114, 10826-10834. 